\title{
Multi-tier Analysis of SiC Breaches in Safety-Tested AGR-1 TRISO Fuel Particles
}

\author{
T.J. Gerczak ${ }^{1}$, J.D. Hunn ${ }^{1}$, C.A. Baldwin ${ }^{1}$, R.N. Morris ${ }^{1}$, F.C. Montgomery ${ }^{1}$, C.M. Silva ${ }^{1}$, and P.A. \\ Demkowicz $^{2}$ \\ 1. Oak Ridge National Laboratory, Oak Ridge, TN, USA \\ 2. Idaho National Laboratory, Idaho Falls, ID, USA
}

Tristructural isotropic (TRISO) coated particle fuel development is being supported by the US Department of Energy, Office of Nuclear Energy. The development plan includes a series of irradiations to qualify TRISO fuel. The first irradiation, AGR-1, included fuel fabricated at Oak Ridge National Laboratory (ORNL) and irradiated in the Advanced Test Reactor at the Idaho National Laboratory (INL). The TRISO fuel design consisted of a uranium carbide/uranium oxide kernel surrounded by concentric coating layers of carbonaceous buffer, inner pyrolitic carbon (IPyC), silicon carbide (SiC), and outer pyrolitic carbon (OPyC). Particles were then over-coated with carbonaceous matrix material and pressed into a cylindrical compact, with each compact containing greater than 4100 TRISO particles. A total of 72 compacts were included in AGR-1 and the irradiation was completed in November 2009 after $\sim 620$ effective full power days [1].

Post irradiation examination (PIE) of AGR-1 is underway at both ORNL and INL. The focus of the PIE is to understand fuel behavior in the as-irradiated state and after compact safety testing at elevated temperatures to simulate accident conditions. Safety testing was conducted in the ORNL Core Conduction Cooldown Test Facility in a specially designed furnace fitted with real-time fission gas monitoring and an exchangeable cold finger to measure release of volatile fission products (FP) during heating [2]. Compact 3-3-1 was subjected to safety testing conditions of $1700^{\circ} \mathrm{C}$ for 300 hours. Very low levels of ${ }^{85} \mathrm{Kr}$ release indicated no complete layer failures, but were accompanied by elevated levels of cesium release indicative of a particle(s) with a $\mathrm{SiC}$ breach and intact IPyC and/or OPyC layers. Further analysis of these anomalous particles provides insights on TRISO failure mechanisms and FP release pathways; however, continued analysis requires that the specific particles first be identified and isolated from other particles in the compact, followed by detailed microanalysis of the SiC breach.

Compact 3-3-1 was electrolytically deconsolidated in nitric acid after safety testing, liberating individual particles. The FP retention behavior for all particles was evaluated using the Advanced Irradiated Microsphere Gamma Analyzer (Advanced-IMGA), which measures the activity of key isotopes by acquiring a unique gamma-ray spectrum for each particle. FP retention behavior is determined by calculating the ratio of the activity of key volatile isotopes to the activity of less-mobile isotopes, e.g. ${ }^{137} \mathrm{Cs}$ to ${ }^{144} \mathrm{Ce}$. Abnormal FP retention behavior reflects the integrity of the TRISO coating layers, as a primary role of the TRISO coatings is to serve as a FP barrier. The Advanced-IMGA was designed to measure a large number of particles and differentiate between particles with varying retention behaviors via an automated process, which allows for thousands of particles to be analyzed and sorted remotely [2]. Advanced-IMGA analysis of Compact 3-3-1 was performed on 4107 particles, where two particles with abnormally low cesium inventories, as shown in Figure 1a, were successfully identified and isolated.

Micro-computed x-ray tomography (micro-XCT) was performed on the two low cesium particles identified by Advanced-IMGA. Micro-XCT provides non-destructive three-dimensional analysis of the 
internal particle microstructure with $\sim 1 \mu \eta \eta$ spatial resolution. This allows for identification and targeting of specific anomalies without disrupting individual layers. Micro-XCT was performed on irradiated TRISO particles using a system designed by ORNL and Xradia based on the Xradia MicroXCT-400, which incorporates a lead-lined stainless steel shielded enclosure to accommodate high activity samples. Micro-XCT indicated localized $\mu$ in-scale breaches in the SiC layer in the low cesium particles, as shown in Figure $\mathrm{lb}$. The SiC breaches were identified to have initiated at IPyC fractures.

The orientations of the SiC breaches determined by micro-XCT were used as a guide for targeting the features for Scanning Electron Microscopy (SEM) analysis, where SEM analysis provides opportunity for improved resolution and determination of elemental information. The buried state of the SiC breaches and two-dimensional nature of standard materialographic sample preparation, limit the probability of successfully producing a sample containing the feature of interest without the additional help of the initial three-dimensional imaging. The difficulty of remote sample preparation performed in a hot cell exacerbates this challenge. Targeting is achieved by orienting the particle, per the micro-XCT analysis, in an aluminum mount such that the breach will appear in cross-section as material is removed. Tomographic slices obtained by micro-XCT serve as a guide during sample preparation, and are compared to periodic optical imaging so that particles are ground to an appropriate depth. Figure lc shows the SEM cross-section obtained by this sample preparation methodology; the analysis indicates FP build-up and interaction at the SiC breach. This analysis also provides an opportunity for additional advanced microscopy through coupled focused ion beam sample preparation and transmission electron microscopy analysis of the features of interest.

The approach demonstrated here shows how compacts that release FPs either during irradiation or safety testing can be identified and specific particles that exhibit anomalous behavior can be isolated from among several thousand particles within the fuel compact. After isolation, coating layers can be characterized to determine the nature of the coating failures and observe the migration of FPs. The use of non-destructive techniques allows for efficient, comprehensive analysis and targeting of the specific regions within the particle associated with coating layer failures. Analysis of the anomalous particles contributes to the understanding of fuel performance and fuel behavior under accident conditions.

\section{References:}

[1] B.P. Collin, “AGR-1 irradiation test final as-run report,” INL/EXT-10-18097, Rev. 1, INL (2012).

[2] C.A. Baldwin et al., Nucl. Eng. and Des., (2013), http://dx.doi.Org/10.1016/j.nucengdes.2013.11.021.

[3] This work was supported by the U.S. Department of Energy, Office of Nuclear Energy.

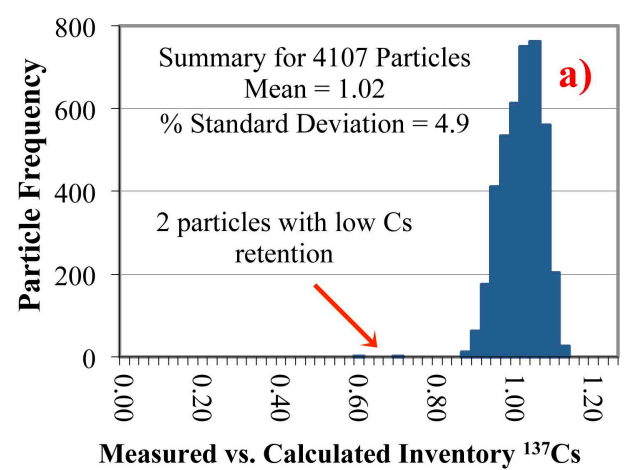

Figure 1. Example of multi-level analysis for Compact 331, (a) identifying ${ }^{137}$ Cs inventory by Advanced IMGA, (b) identifying layer defects by XT, and (c) site-specific SEM analysis of SiC breach. 